\title{
Some extensions Hardy integral inequalities and their analogues on finite interval
}

\author{
Artion Kashuri, Rozana Liko \\ Department of Mathematics, Faculty of Technical Science, \\ University “Ismail Qemali”, Vlora. Albania \\ artionkashuri@gmail.com, rozanaliko86@gmail.com
}

\section{ABSTRACT}

The aim of this paper is to give some extensions Hardy integral inequalities for sum and product of several functions and their analogues inequalities on finite interval. Some direct consequences are established. Also a partial answer of an open problem posed by Sroysang is obtained.

\section{Indexing terms/Keywords}

Hardy's integral inequality; Levinson's integral inequality.

\section{Academic Discipline And Sub-Disciplines}

Mathematical Analysis, Mathematical Inequalities, Applied Mathematics.

\section{SUBJECT CLASSIFICATION}

2010Mathematics Subject Classification: 26D15.

\section{INTRODUCTION}

In 1920, Hardy (see [1]) presented the following inequality

$$
\int_{0}^{+\infty}\left(\frac{F(x)}{x}\right)^{p} d x \leq\left(\frac{p}{p-1}\right)^{p} \int_{0}^{+\infty} f^{p}(x) d x
$$

where $f \geq 0, p>1$ and

$$
F(x)=\int_{0}^{x} f(t) d t
$$

The constant $\left(\frac{\mathrm{p}}{\mathrm{p}-1}\right)^{\mathrm{p}}$ is the best possible. This inequality is important in mathematical analysis and its applications. In 1964, Levinson (see [2], [3]) presented the following analogue of Hardy's integral inequality on finite interval[a, b]

$$
\int_{a}^{b}\left(\frac{F(x)}{x}\right)^{p} d x \leq\left(\frac{p}{p-1}\right)^{p} \int_{a}^{b} f^{p}(x) d x
$$

where $0<\mathrm{a}<\mathrm{b}<+\infty$, f $\geq 0, \mathrm{p}>1$ and

$$
F(x)=\int_{0}^{x} f(t) d t
$$

In 2006, Bougoffa (see [4]) prove the following theorem about Hardy's integral inequality for several functions.

Theorem 1. 1. Letf $_{1}, f_{2}, \cdots, f_{i}$ be nonnegative integrable functions. DefineF $(x)=\int_{a}^{x} f_{k}(t) d t$, where $k=1,2, \cdots, i$.

Then for $\mathrm{p}>1$, we have

$$
\int_{0}^{+\infty}\left(\frac{\mathrm{F}_{1}(\mathrm{x}) \mathrm{F}_{2}(\mathrm{x}) \cdots \mathrm{F}_{\mathrm{i}}(\mathrm{x})}{\mathrm{x}^{\mathrm{i}}}\right)^{\frac{\mathrm{p}}{\mathrm{i}}} \mathrm{dx} \leq\left(\frac{\mathrm{p}}{\mathrm{p}-1}\right)^{\mathrm{p}} \int_{0}^{+\infty}\left(\mathrm{f}_{1}(\mathrm{x})+\mathrm{f}_{2}(\mathrm{x})+\cdots+\mathrm{f}_{\mathrm{i}}(\mathrm{x})\right)^{\mathrm{p}} \mathrm{dx} .
$$

The main purpose of this paper is to give several extensions Hardy integral inequalities for sum and product of several functions and their analogues inequalities on finite interval $[a, b]$ using Levinson's integral inequality (1.2). Some direct consequences are established. Special case obtained give a partial answer of an open problem posed by Sroysang in 2014(see [3]).

\section{MAIN RESULTS}

Throughout this section, functions are assumed to be integrable. Let 


$$
F(x)=\int_{0}^{x}\left(\sum_{i=1}^{n} f_{i}(t)\right) d t, \quad G(x)=\int_{0}^{x}\left(\sum_{i=1}^{m} g_{i}(t)\right) d t .
$$

Now, we are in position to prove the following theorem for sum of several functions.

Theorem 2. 1. Let $0 \leq \sum_{\mathrm{i}=1}^{\mathrm{n}} \mathrm{f}_{\mathrm{i}} \leq \sum_{\mathrm{i}=1}^{\mathrm{m}} \mathrm{g}_{\mathrm{i}}$ and $\sum_{\mathrm{i}=1}^{\mathrm{m}} \mathrm{g}_{\mathrm{i}}$ nonidentically zero, for all $\mathrm{x} \in[0,+\infty$ [. Define $\mathrm{F}(\mathrm{x})$, $\mathrm{G}(\mathrm{x})$ by $(2.1)$. Then

$$
\int_{0}^{+\infty} \frac{\mathrm{F}^{\mathrm{p}}(\mathrm{x})}{\mathrm{G}^{\mathrm{q}}(\mathrm{x})} \mathrm{dx} \leq\left(\frac{\mathrm{p}-\mathrm{q}}{\mathrm{p}-\mathrm{q}-1}\right)^{\mathrm{p}-\mathrm{q}} \int_{0}^{+\infty} \varphi^{\mathrm{p}-\mathrm{q}}(\mathrm{x}) \mathrm{dx},
$$

where $\varphi(x)=x\left(\sum_{i=1}^{m} g_{i}(x)\right)+G(x), p-q>1, p>0$

Proof.Let $0 \leq \sum_{i=1}^{n} f_{i} \leq \sum_{i=1}^{m} g_{i}$ and $\sum_{i=1}^{m} g_{i}$ nonidentically zero, for all $x \in\left[0,+\infty\left[\right.\right.$. Then, for all $p>0,0 \leq F^{p}(x) \leq G^{p}(x)$. By using inequality (1.1) we get

$$
\begin{array}{r}
\int_{0}^{+\infty} \frac{\mathrm{F}^{\mathrm{p}}(\mathrm{x})}{\mathrm{G}^{\mathrm{q}}(\mathrm{x})} \mathrm{dx} \leq \int_{0}^{+\infty} \frac{\mathrm{G}^{\mathrm{p}}(\mathrm{x})}{\mathrm{G}^{\mathrm{q}}(\mathrm{x})} \mathrm{dx}=\int_{0}^{+\infty}\left(\frac{\mathrm{G}^{*}(\mathrm{x})}{\mathrm{x}}\right)^{\mathrm{p}-\mathrm{q}} \mathrm{dx} \leq\left(\frac{\mathrm{p}-\mathrm{q}}{\mathrm{p}-\mathrm{q}-1}\right)^{\mathrm{p}-\mathrm{q}} \int_{0}^{+\infty} \varphi^{\mathrm{p}-\mathrm{q}}(\mathrm{x}) \mathrm{dx}, \\
\text { where }^{*}(\mathrm{x})=\mathrm{xG}(\mathrm{x}) \text { and } \varphi(\mathrm{x})=\mathrm{x}\left(\sum_{\mathrm{i}=1}^{\mathrm{m}} \mathrm{g}_{\mathrm{i}}(\mathrm{x})\right)+\mathrm{G}(\mathrm{x}), \mathrm{p}-\mathrm{q}>1, \mathrm{p}>0 .
\end{array}
$$

Analogue of Theorem 2.1 on finite interval $[a, b], 0<a<b<+\infty$ is as follows.

Theorem 2. 2. Let $0 \leq \sum_{\mathrm{i}=1}^{\mathrm{n}} \mathrm{f}_{\mathrm{i}} \leq \sum_{\mathrm{i}=1}^{\mathrm{m}} \mathrm{g}_{\mathrm{i}}$ and $\sum_{\mathrm{i}=1}^{\mathrm{m}} \mathrm{g}_{\mathrm{i}}$ nonidentically zero, for all $\mathrm{x} \in[\mathrm{a}, \mathrm{b}], 0<\mathrm{a}<\mathrm{b}<+\infty$. Define $\mathrm{F}(\mathrm{x})$, $\mathrm{G}(\mathrm{x})$ by (2.1). Then

$$
\int_{a}^{b} \frac{F^{p}(x)}{G^{q}(x)} d x \leq\left(\frac{p-q}{p-q-1}\right)^{p-q} \int_{a}^{b} \varphi^{p-q}(x) d x
$$

where $\varphi(\mathrm{x})=\mathrm{x}\left(\sum_{\mathrm{i}=1}^{\mathrm{m}} \mathrm{g}_{\mathrm{i}}(\mathrm{x})\right)+\mathrm{G}(\mathrm{x}), \mathrm{p}-\mathrm{q}>1, \mathrm{p}>0$.

Proof.Let $0 \leq \sum_{\mathrm{i}=1}^{\mathrm{n}} \mathrm{f}_{\mathrm{i}} \leq \sum_{\mathrm{i}=1}^{\mathrm{m}} \mathrm{g}_{\mathrm{i}}$ and $\sum_{\mathrm{i}=1}^{\mathrm{m}} \mathrm{g}_{\mathrm{i}}$ nonidentically zero, for all $\mathrm{x} \in[\mathrm{a}, \mathrm{b}], 0<\mathrm{a}<\mathrm{b}<+\infty$. Then, for all $\mathrm{p}>0,0 \leq$ $\mathrm{F}^{\mathrm{p}}(\mathrm{x}) \leq \mathrm{G}^{\mathrm{p}}(\mathrm{x})$. By using inequality (1.2) we get

$$
\begin{aligned}
\int_{a}^{b} \frac{F^{p}(x)}{G^{q}(x)} d x \leq \int_{a}^{b} \frac{G^{p}(x)}{G^{q}(x)} d x=\int_{a}^{b}\left(\frac{G^{*}(x)}{x}\right)^{p-q} d x \leq\left(\frac{p-q}{p-q-1}\right)^{p-q} \int_{a}^{b} \varphi^{p-q}(x) d x, \\
\text { where } G^{*}(x)=x G(x) \text { and } \varphi(x)=x\left(\sum_{i=1}^{m} g_{i}(x)\right)+G(x), p-q>1, p>0 .
\end{aligned}
$$

Let

$$
F_{k}(x)=\int_{0}^{x} f_{k}(t) d t, \quad G_{k}(x)=\int_{0}^{x} g_{k}(t) d t \text { and } k=1,2, \cdots, n .
$$

Now, we are in position to prove the following theorem for product of several functions.

Theorem 2. 3. Let $0 \leq \mathrm{f}_{\mathrm{k}} \leq \mathrm{g}_{\mathrm{k}}$ for all $\mathrm{k}=1,2, \cdots, \mathrm{n}, \mathrm{g}_{\mathrm{k}}$ nonidentically zero for all for all $\mathrm{x} \in\left[0,+\infty\left[\right.\right.$. Define $\mathrm{F}_{\mathrm{k}}(\mathrm{x}), \mathrm{G}_{\mathrm{k}}(\mathrm{x})$ by (2.4). Then

$$
\int_{0}^{+\infty} \frac{\left(\prod_{\mathrm{k}=1}^{\mathrm{n}} \mathrm{F}_{\mathrm{k}}(\mathrm{x})\right)^{\mathrm{p}}}{\left(\prod_{\mathrm{k}=1}^{\mathrm{n}} \mathrm{G}_{\mathrm{k}}(\mathrm{x})\right)^{\mathrm{q}}} \mathrm{dx} \leq\left(\frac{\mathrm{p}-\mathrm{q}}{\mathrm{n}(\mathrm{p}-\mathrm{q}-1)}\right)^{\mathrm{p}-\mathrm{q}} \int_{0}^{+\infty}\left(\sum_{\mathrm{k}=1}^{\mathrm{n}} \varphi_{\mathrm{k}}(\mathrm{x})\right)^{\mathrm{p}-\mathrm{q}} \mathrm{dx},(2.5)
$$

where $\varphi_{\mathrm{k}}(\mathrm{x})=\operatorname{nxg}_{\mathrm{k}}(\mathrm{x}) \mathrm{G}_{\mathrm{k}}^{\mathrm{n}-1}(\mathrm{x})+\mathrm{G}_{\mathrm{k}}^{\mathrm{n}}(\mathrm{x})$ for all $\mathrm{k}=1,2, \cdots, \mathrm{n}, \mathrm{p}-\mathrm{q}>1, \mathrm{p}>0$.

Proof.Let $0 \leq \mathrm{f}_{\mathrm{k}} \leq \mathrm{g}_{\mathrm{k}}$ for all $\mathrm{k}=1,2, \cdots, \mathrm{n}$, $\mathrm{g}_{\mathrm{k}}$ nonidentically zero for all for all $\mathrm{x} \in[0,+\infty[$. Then, for all $\mathrm{p}>0,0 \leq$ $\left(\prod_{\mathrm{k}=1}^{\mathrm{n}} \mathrm{F}_{\mathrm{k}}(\mathrm{x})\right)^{\mathrm{p}} \leq\left(\prod_{\mathrm{k}=1}^{\mathrm{n}} \mathrm{G}_{\mathrm{k}}(\mathrm{x})\right)^{\mathrm{p}}$. By using Theorem 1.1 we get

$$
\begin{aligned}
& \int_{0}^{+\infty} \frac{\left(\prod_{k=1}^{\mathrm{n}} \mathrm{F}_{\mathrm{k}}(\mathrm{x})\right)^{\mathrm{p}}}{\left(\prod_{\mathrm{k}=1}^{\mathrm{n}} \mathrm{G}_{\mathrm{k}}(\mathrm{x})\right)^{\mathrm{q}}} \mathrm{dx} \leq \int_{0}^{+\infty} \frac{\left(\prod_{\mathrm{k}=1}^{\mathrm{n}} \mathrm{G}_{\mathrm{k}}(\mathrm{x})\right)^{\mathrm{p}}}{\left(\prod_{\mathrm{k}=1}^{\mathrm{n}} \mathrm{G}_{\mathrm{k}}(\mathrm{x})\right)^{\mathrm{q}}} \mathrm{dx}=\int_{0}^{+\infty}\left(\prod_{\mathrm{k}=1}^{\mathrm{n}} \Psi_{\mathrm{k}}(\mathrm{x})\right)^{\frac{\mathrm{p}-\mathrm{q}}{\mathrm{n}}} \mathrm{dx} \\
& =\int_{0}^{+\infty}\left(\frac{\prod_{\mathrm{k}=1}^{\mathrm{n}} \Phi_{\mathrm{k}}(\mathrm{x})}{\mathrm{x}^{\mathrm{n}}}\right)^{\frac{\mathrm{p}-\mathrm{q}}{\mathrm{n}}} \mathrm{dx} \leq\left(\frac{\mathrm{p}-\mathrm{q}}{\mathrm{n}(\mathrm{p}-\mathrm{q}-1)}\right)^{\mathrm{p}-\mathrm{q}} \int_{0}^{+\infty}\left(\sum_{\mathrm{k}=1}^{\mathrm{n}} \varphi_{\mathrm{k}}(\mathrm{x})\right)^{\mathrm{p}-\mathrm{q}} \mathrm{dx},
\end{aligned}
$$


where $\Phi_{\mathrm{k}}(\mathrm{x})=\mathrm{x} \Psi_{\mathrm{k}}(\mathrm{x})=\mathrm{xG}_{\mathrm{k}}^{\mathrm{n}}(\mathrm{x})=\int_{0}^{\mathrm{x}} \varphi_{\mathrm{k}}(\mathrm{t}) \mathrm{dt}$, for all $\mathrm{k}=1,2, \cdots, \mathrm{n}$.

After differentiation we get $\varphi_{k}(x)=n x g_{k}(x) G_{k}^{n-1}(x)+G_{k}^{n}(x)$ for all $k=1,2, \cdots, n, p-q>1, p>0$.

Analogue of Theorem 2.3 on finite interval $[a, b], 0<a<b<+\infty$ is as follows.

Theorem 2. 4. Let $0 \leq f_{k} \leq g_{k}$ for all $k=1,2, \cdots, n, g_{k}$ nonidentically zero for all for all $x \in[a, b], 0<a<b<+\infty$. Define $F_{k}(x), G_{k}(x)$ by $(2.4)$. Then

$$
\int_{a}^{b} \frac{\left(\prod_{k=1}^{n} F_{k}(x)\right)^{p}}{\left(\prod_{k=1}^{n} G_{k}(x)\right)^{q}} d x \leq\left(\frac{p-q}{n(p-q-1)}\right)^{p-q} \int_{a}^{b}\left(\sum_{k=1}^{n} \varphi_{k}(x)\right)^{p-q} d x,(2.6)
$$

where $\varphi_{k}(x)=n \times g_{k}(x) G_{k}^{n-1}(x)+G_{k}^{n}(x)$ for all $k=1,2, \cdots, n, p-q>1, p>0$.

Proof.Let $0 \leq f_{k} \leq g_{k}$ for all $k=1,2, \cdots, n, g_{k}$ nonidentically zero for all for all $x \in[a, b], 0<a<b<+\infty$. Then, for all $p>0,0 \leq\left(\prod_{k=1}^{n} F_{k}(x)\right)^{p} \leq\left(\prod_{k=1}^{n} G_{k}(x)\right)^{p}$.By using Theorem 1.1and inequality (1.2)we get

$$
\begin{aligned}
& \int_{a}^{b} \frac{\left(\prod_{k=1}^{n} F_{k}(x)\right)^{p}}{\left(\prod_{k=1}^{n} G_{k}(x)\right)^{q}} d x \leq \int_{a}^{b} \frac{\left(\prod_{k=1}^{n} G_{k}(x)\right)^{p}}{\left(\prod_{k=1}^{n} G_{k}(x)\right)^{q}} d x=\int_{a}^{b}\left(\prod_{k=1}^{n} \Psi_{k}(x)\right)^{\frac{p-q}{n}} d x \\
& =\int_{a}^{b}\left(\frac{\prod_{k=1}^{n} \Phi_{k}(x)}{x^{n}}\right)^{\frac{p-q}{n}} d x \leq\left(\frac{p-q}{n(p-q-1)}\right)^{p-q} \int_{a}^{b}\left(\sum_{k=1}^{n} \varphi_{k}(x)\right)^{p-q} d x,
\end{aligned}
$$

where $\Phi_{k}(x)=x \Psi_{k}(x)=x G_{k}^{n}(x)=\int_{0}^{x} \varphi_{k}(t) d t$, for all $k=1,2, \cdots, n$.

After differentiation we get $\varphi_{k}(x)=n x g_{k}(x) G_{k}^{n-1}(x)+G_{k}^{n}(x)$ for all $k=1,2, \cdots, n, p-q>1, p>0$.

Remark2. 5.Special case: Theorem 2.3 for $k=1$ has the following form

$$
\int_{0}^{+\infty} \frac{F_{1}{ }^{p}(x)}{G_{1}{ }^{q}(x)} d x \leq\left(\frac{p-q}{p-q-1}\right)^{p-q} \int_{0}^{+\infty} \varphi_{1}^{p-q}(x) d x
$$

where $\varphi_{1}(x)=x g_{1}(x)+G_{1}(x), p-q>1, p>0$.

This is a partial answer of an open problem posed by Sroysang in 2014 (see [3])

Analogue inequality on finite interval $[a, b], 0<a<b<+\infty$, is as follows.

$$
\int_{a}^{b} \frac{F_{1}^{p}(x)}{G_{1}{ }^{q}(x)} d x \leq\left(\frac{p-q}{p-q-1}\right)^{p-q} \int_{a}^{b} \varphi_{1}^{p-q}(x) d x,
$$

where $\varphi_{1}(x)=x g_{1}(x)+G_{1}(x), p-q>1, p>0$.Use Theorem 2.4 for $k=1$.

Next, we give some direct consequences of Theorems 2.1, 2.2, 2.3and 2.4.

\section{APPLICATIONS}

Corollary3. 1.Let $0 \leq \sum_{i=1}^{n} f_{i} \leq \sum_{i=1}^{m} g_{i}$ and $\sum_{i=1}^{m} g_{i}$ nonidentically zero, for all $x \in[0,+\infty[$. Define $F(x), G(x)$ by $(2.1)$. Then

$$
\int_{0}^{+\infty}(F(x) G(x))^{p} d x \leq\left(\frac{2 p}{2 p-1}\right)^{2 p} \int_{0}^{+\infty} \varphi^{2 p}(x) d x
$$

where $\varphi(x)=x\left(\sum_{i=1}^{m} g_{i}(x)\right)+G(x)$ and $p>\frac{1}{2}$.

Proof.Let $q=-p$ and use Theorem 2.1.

Analogue inequality of Corollary 3.1 on finite interval $[a, b]$, is as follows.

Corollary3. 2. Let $0 \leq \sum_{i=1}^{n} f_{i} \leq \sum_{i=1}^{m} g_{i}$ and $\sum_{i=1}^{m} g_{i}$ nonidentically zero, for all $x \in[a, b], 0<a<b<+\infty$. Define $F(\mathrm{x}), G(x)$ by (2.1). Then

$$
\int_{a}^{b}(F(x) G(x))^{p} d x \leq\left(\frac{2 p}{2 p-1}\right)^{2 p} \int_{a}^{b} \varphi^{2 p}(x) d x,(3.2)
$$


where $\varphi(x)=x\left(\sum_{i=1}^{m} g_{i}(x)\right)+G(x)$ and $p>\frac{1}{2}$.

Proof.Let $q=-p$ and use Theorem 2.2.

Corollary3. 3. Let $0 \leq f_{k} \leq g_{k}$ for all $k=1,2, \cdots, n, g_{k}$ nonidentically zero for all for all $x \in\left[0,+\infty\left[\right.\right.$. Define $F_{k}(x), G_{k}(x)$ by (2.4). Then

$$
\int_{0}^{+\infty}\left(\prod_{k=1}^{n} \mathrm{~F}_{k}(x) G_{k}(x)\right)^{p} d x \leq\left(\frac{2 p}{n(2 p-1)}\right)^{2 p} \int_{0}^{+\infty}\left(\sum_{k=1}^{n} \varphi_{k}(x)\right)^{2 p} d x
$$

where $\varphi_{k}(x)=n x g_{k}(x) G_{k}^{n-1}(x)+G_{k}^{n}(x)$ for all $k=1,2, \cdots, n$ and $p>\frac{1}{2}$.

\section{Proof.Let $q=-p$ and use Theorem 2.3.}

Corollary3. 4.Let $f_{k}>0$ for all $k=1,2, \cdots, n$, and for all $x \in\left[0,+\infty\left[\right.\right.$. Define $F_{k}(x)$ by (2.4). Then

$$
\int_{0}^{+\infty}\left(\prod_{k=1}^{n} F_{k}(x)\right)^{2 p} d x \leq\left(\frac{2 p}{n(2 p-1)}\right)^{2 p} \int_{0}^{+\infty}\left(\sum_{k=1}^{n} \varphi_{k}(x)\right)^{2 p} d x,(3.4)
$$

where $\varphi_{k}(x)=n x f_{k}(x) F_{k}^{n-1}(x)+F_{k}^{n}(x)$ for all $k=1,2, \cdots, n$ and $p>\frac{1}{2}$

Proof.Let $f_{k}=g_{k}$ for all $k=1,2, \cdots, n$ and use Corollary3.3.

Corollary3. 5. Let $0 \leq f_{k}=f \leq g=g_{k}$ for all $k=1,2, \cdots, n, g$ nonidentically zero for all for all $x \in[0,+\infty$ [.Define

$$
F(x)=\int_{0}^{x} f(t) d t, \quad G(x)=\int_{0}^{x} g(t) d t .
$$

Then

$$
\int_{0}^{+\infty}\left(\frac{F^{p}(x)}{G^{q}(x)}\right)^{n} d x \leq\left(\frac{p-q}{p-q-1}\right)^{p-q} \int_{0}^{+\infty} \varphi^{p-q}(x) d x
$$

where $\varphi(x)=n x g(x) G^{n-1}(x)+G^{n}(x), p-q>1, p>0$.

Proof.Let $\leq \leq f_{k}=f \leq g=g_{k}$ for all $k=1,2, \cdots, n, g$ nonidentically zero for all for all $x \in\left[0,+\infty\left[\right.\right.$. Then $F_{k}(x)=F(x)$ and $G_{k}(x)=G(x)$ for all $k=1,2, \cdots, n$.Using Theorem 2.3, inequality (3.5) follows.

Remark3. 6. Inequality (3.5) is a generalization of inequality (2.7).

Analogues inequalities for Corollaries $3.3,3.4$ and 3.5 on finite interval are as follows.

Corollary3. 7.Let $0 \leq f_{k} \leq g_{k}$ for all $k=1,2, \cdots, n, g_{k}$ nonidentically zero for all for all $x \in[a, b], 0<a<b<+\infty$. Define $F_{k}(x), G_{k}(x)$ by $(2.4)$. Then

$$
\int_{a}^{b}\left(\prod_{k=1}^{n} F_{k}(x) G_{k}(x)\right)^{p} d x \leq\left(\frac{2 p}{n(2 p-1)}\right)^{2 p} \int_{a}^{b}\left(\sum_{k=1}^{n} \varphi_{k}(x)\right)^{2 p} d x,(3.6)
$$

where $\varphi_{k}(x)=n x g_{k}(x) G_{k}^{n-1}(x)+G_{k}^{n}(x)$ for all $k=1,2, \cdots$, nand $p>\frac{1}{2}$

Proof.Let $q=-p$ and use Theorem 2.4.

Corollary3. 8. Let $f_{k}>0$ for all $k=1,2, \cdots, n$ and for all $x \in[a, b], 0<a<b<+\infty$. Define $F_{k}(x)$ by (2.4). Then

$$
\int_{a}^{b}\left(\prod_{k=1}^{n} F_{k}(x)\right)^{2 p} d x \leq\left(\frac{2 p}{n(2 p-1)}\right)^{2 p} \int_{a}^{b}\left(\sum_{k=1}^{n} \varphi_{k}(x)\right)^{2 p} d x,(3.7)
$$

where $\varphi_{k}(x)=n x f_{k}(x) F_{k}^{n-1}(x)+F_{k}^{n}(x)$ for all $k=1,2, \cdots, n$ and $p>\frac{1}{2}$.

Proof.Let $f_{k}=g_{k}$ for all $k=1,2, \cdots, n$ and use Corollary3.7.

Corollary3. 9. Let $0 \leq f_{k}=f \leq g=g_{k}$ for all $k=1,2, \cdots, n$, gnonidentically zero for all $x \in[a, b], 0<a<b<+\infty$.Define

$$
F(x)=\int_{0}^{x} f(t) d t, \quad G(x)=\int_{0}^{x} g(t) d t .
$$

Then 


$$
\int_{a}^{b}\left(\frac{F^{p}(x)}{G^{q}(x)}\right)^{n} d x \leq\left(\frac{p-q}{p-q-1}\right)^{p-\mathrm{q}} \int_{\mathrm{a}}^{\mathrm{b}} \varphi^{\mathrm{p}-\mathrm{q}}(\mathrm{x}) \mathrm{dx},(3.8)
$$

where $\varphi(x)=n x g(x) G^{n-1}(x)+G^{n}(x), p-q>1, p>0$.

Proof.Let0 $\leq \mathrm{f}_{\mathrm{k}}=\mathrm{f} \leq \mathrm{g}=\mathrm{g}_{\mathrm{k}}$ for all $\mathrm{k}=1,2, \cdots, \mathrm{n}$, gnonidentically zero for all $\mathrm{x} \in[\mathrm{a}, \mathrm{b}], 0<\mathrm{a}<\mathrm{b}<+\infty$.Then $\mathrm{F}_{\mathrm{k}}(\mathrm{x})=\mathrm{F}(\mathrm{x})$ and $\mathrm{G}_{\mathrm{k}}(\mathrm{x})=\mathrm{G}(\mathrm{x})$ for all $\mathrm{k}=1,2, \cdots, \mathrm{n}$.Using Theorem 2.4, inequality (3.8)holds.

\section{ACKNOWLEDGMENTS}

We thank anonymous referee for his/her valuable suggestion regarding the manuscript.

\section{REFERENCES}

1. G. H. Hardy Notes on a theorem of Hilbert Math. Z.,6, (1920), $314-317$.

2. S.Wu, B. Sroysang, S. Li A further generalization of certain integral inequalities similar toHardy's inequality J. Nonlinear Sci. Appl., 9, (2016), 1093 - 1102.

3. B. Sroysang More on some Hardy type integral inequalitiesJMI, J. Math. Inequal.,8, (2014), $497-501$.

4. L. Bougoffa On Minkowski and Hardy Integral InequalitiesJ. Ineq. Pure and Appl. Math.,7(2)Art. 60, (2006).

\section{Author' biography with Photo}

Ph.D Artion KASHURI

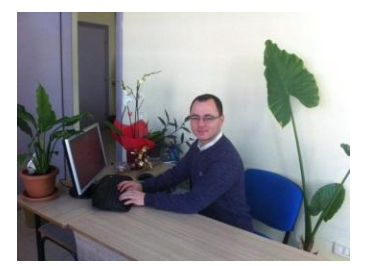

Lector, University “Ismail Qemali”, Vlora, Albania 2009 - 2016

Ph.D Candidate Rozana LIKO

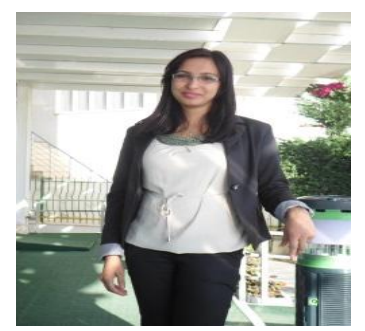

Lecturer, University “Ismail Qemali”, Vlora, Albania 2009 - 2016 